\title{
Toxicidade aguda (LC50) e efeitos \\ comportamentais e morfológicos de formulado comercial com princípio ativo glifosato em girinos de Physalaemus cuvieri (Anura, Leptodactylidae) e Rhinella icterica (Anura, Bufonidae)
}

Acute Toxicity (CL5O) and behavioral and morphological effects of a commercial formulation with glyphosate active ingredient in tadpoles of Physalaemus cuvieri (Anura, Leptodactylidae) and Rhinella icterica (Anura, Bufonidae)

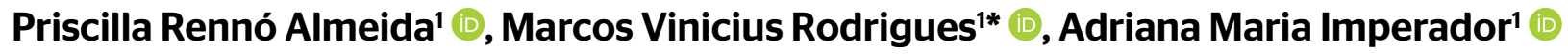

\section{RESUMO}

O declínio de anfíbios no mundo está cada vez maior e a perda de riqueza, muitas vezes, está associada a áreas agrícolas. O uso de agrotóxicos, em especial daqueles à base de glifosato, é cada vez maior e novas formulações estão sendo desenvolvidas. O presente trabalho avaliou a toxicidade aguda de um composto comercial com o princípio ativo glifosato e os efeitos comportamentais e morfológicos em girinos de Physalaemus cuvieri e Rhinella icterica. O primeiro mostrou-se mais sensível em relação ao formulado comercial do que o segundo e não houve diferenças significativas em relação às mudanças comportamentais e o aumento das concentrações do formulado entre as espécies, porém houve alterações morfológicas de peso e comprimento dos girinos de ambas espécies.

Palavras-chave: glifosato; toxicidade aguda; Physalaemus cuvieri; Rhinella icterica; comportamento; malformações.

\begin{abstract}
The decline of amphibians in the world is increasing and the loss of wealth is often associated with agricultural areas. The use of pesticides, especially those based on glyphosate is increasing and new formulations are being developed. This study evaluated the acute toxicity of a commercial compound with glyphosate and behavioral and morphological effects on Physalaemus cuvieri and Rhinella icterica tadpoles; the former was more sensitive to the commercially formulated compound than R. icterica and there were no significant differences in relation to behavioral changes and increase compound concentrations among species, but there were morphological changes of weight and length of tadpoles of both species.
\end{abstract}

Keywords: glyphosate; acute toxicity; Physalaemus cuvieri; Rhinella icterica: behavior; malformations.

\section{INTRODUÇÃO}

O desenvolvimento acelerado da agricultura tem levado ao aumento do uso de agrotóxicos. De acordo com Gasques et al. (2012), nos últimos 37 anos, o produto agrícola, no Brasil, cresceu a uma taxa média anual de $3,77 \%$. A quantidade de poluentes despejados nos ambientes aquáticos vem crescendo a cada ano, interagindo de forma adversa com o meio (GUIMARÃES; LACAVA; MAGALHÃES, 2004).
Os agrotóxicos são poluentes ambientais que podem ser uma importante causa de mortalidade da fauna e da flora, pois vários declínios estão associados a áreas agrícolas e adjacentes (RELYEA, 2005) e, quando utilizados para combater pragas específicas ou pragas de uma forma geral, sua utilização acaba afetando outros organismos que não são o alvo do produto (RELYEA; JONES, 2009).

O glifosato é o princípio ativo mais utilizado em agrotóxicos no mundo devido a sua alta eficácia no controle de plantas daninhas na agricultura (HOWE et al., 2004; RELYEA; JONES, 2009). Em plantas e 
alguns microrganismos, ele age como inibidor de enzimas essenciais na síntese de aminoácidos necessários à síntese proteica das plantas, causando déficit de aminoácidos aromáticos (ITHO, 2014). Com a redução desses aminoácidos nas plantas, todos os processos que demandam a participação de enzimas e proteínas são diretamente afetados.

Diferentes formulações desse princípio possuem diferentes surfactantes (surface active agent), que atuam como cosolventes que tendem a reduzir a tensão superficial do composto em que estão dissolvidas. Surfactantes são considerados ingredientes inertes e não necessitam de testes ou descrição na formulação do produto. Como consequência, fabricantes constantemente desenvolvem novos surfactantes, o que torna necessários constantes testes de toxicidade em organismos não alvos (RELYEA; JONES, 2009).

A formulação de glifosato mais comumente utilizada emprega o sal de isopropilamina associado ao surfactante polioxietileno amina (POEA) e água. Os solventes utilizados em formulações comerciais podem alterar as propriedades toxicológicas do produto, e alguns surfactantes podem apresentar ações irritativas de importância, que não são resultadas do próprio princípio ativo (ITHO, 2014). Estima-se que o surfactante POEA apresente toxicidade três vezes maior do que o glifosato (AMARANTE JUNIOR et al., 2002).

Os anfíbios são um dos grupos de animais mais ameaçados de extinção em todo o mundo e vêm sofrendo uma queda de riqueza em grandes proporções desde a década de 1980 (IUCN, 2013). São considerados bioindicadores por terem contato com estressores tanto aquáticos (em suas formas larval e adulta) como atmosféricos e do solo (em sua forma adulta) e por possuírem a pele permeável e ovos sem cascas, que ficam diretamente expostos ao solo, à água e à luz solar. Compreendem a maior biomassa de vertebrados e contribuem significativamente para a dinâmica trófica em muitas comunidades - o declínio de anfíbios anuros pode ter impactos importantes sobre outros organismos (BLAUSTEIN; WAKE; SOUSA, 1994; BLAUSTEIN; KIESECKER, 2002).

Os girinos possuem características estruturais específicas que são ausentes nos adultos. $\mathrm{O}$ aparato oral é um exemplo, pois é uma estrutura presente apenas na fase larval dos anuros. Alterações nas estruturas orais podem provocar uma redução na eficiência de forrageamento dos organismos, pois essas estruturas são fundamentais para que os girinos possam se alimentar (MCDIARMID; ALTIG, 1999).

Na metamorfose para a vida adulta, as alterações morfológicas são extremas. Uma das mudanças mais drásticas que ocorrem durante a metamorfose é a diferenciação do trato digestório, com um rearranjo dos órgãos na cavidade visceral. Nesse período, ocorrem acentuada diminuição do intestino e do desenvolvimento do estômago e degeneração da região oral do girino para a formação da boca e o desenvolvimento da língua (BAHIA, 2007).

Transformações químicas, biológicas e físicas de poluentes ambientais podem atingir até os níveis tróficos mais altos, por bioacumulação de alguns elementos, e a agricultura mal manejada influencia na qualidade das águas e, consequentemente, da biota aquática (GUIMARÃES; LACAVA; MAGALHÃES, 2004). Vários fatores são apresentados para explicar o declínio dos anfíbios, como alterações antrópicas, mudanças climáticas e destruição do hábitat. Outras possíveis causas têm sido apontadas, como aumento da exposição à radiação ultravioleta, doenças emergentes, presença de espécies exóticas e exposição a poluentes ambientais (BRUNELLI et al., 2009; DAVID et al., 2012; LAJMANOVICH; SANDOVAL; PELTZER, 2003; RELYEA, 2005).

O impacto de poluentes ambientais na população de anfíbios é influenciado pelas alterações climáticas e modificação do hábitat. As mudanças climáticas afetam os efeitos dos contaminantes, pois, na maioria dos casos, a meia-vida dos poluentes químicos é dependente de vários fatores, como temperatura, $\mathrm{pH}$, incidência de luz, umidade etc., que podem reduzir o impacto do composto químico ou transformar os poluentes em formas mais prejudiciais (HAYES et al., 2010).

O efeito de poluentes em girinos é menos conhecido em relação aos anfíbios adultos (MCDIARMID; ALTIG, 1999). De acordo com Seixas Filho et al. (2003), os girinos, nos estágios menos desenvolvidos, são mais sensíveis do que aqueles em fases mais avançadas, apresentando menor capacidade de ajuste ao meio e não resistindo ao intenso trabalho metabólico.

Muitas espécies usam, em parte de seu ciclo de vida, lagoas temporárias e pequenos cursos d'águas adjacentes às áreas agrícolas, onde os agrotóxicos são aplicados, e, muitas vezes, essas aplicações coincidem com o período reprodutivo e desenvolvimento larval (DAVID et al., 2012; HAYES et al., 2010; HOWE et al., 2004). A contaminação de corpos d'água próximos à área de agricultura geralmente aumenta durante a estação chuvosa, período de reprodução da maioria das espécies de anfíbios. Esses poluentes podem estar dissolvidos na água ou associados ao solo ou a partículas minerais (DAVID et al., 2012; WALKER et al., 2006).

Physalaemus cuvieri é uma espécie de anfíbio anuro da família Leptodactylidae que possui ampla distribuição, sendo conhecida nas regiões Sul, Sudeste, Nordeste e central do Brasil, além de Argentina e Paraguai (MIJARES; RODRIGUES; BALDO, 2010; HERPETO.ORG, 2014). É considerada pouco preocupante em relação à conservação, por causa da sua ampla distribuição geográfica, da tolerância em uma ampla gama de hábitats e regiões e população estável (MIJARES; RODRIGUES; BALDO, 2010). É uma espécie de hábitos noturnos e sua reprodução ocorre em poças, temporárias ou permanentes, geralmente em áreas abertas. Os ovos são depositados em ninhos de espuma flutuante presos à vegetação marginal das poças (CONDEZ; SAWAYA; DIXO, 2009). A espécie Physalaemus cuvieri constitui bom modelo para avaliação da suscetibilidade de espécies nativas por se tratar de uma espécie abundante e de ampla distribuição geográfica no Brasil (SILVA et al., 2013).

Rhinella icterica é uma espécie da família Bufonidae com ampla distribuição, encontrada em mais de um bioma, ocorrendo em áreas florestadas 
e também em fisionomias abertas, desde o Sul e Sudeste do Brasil até o Norte da Argentina e o Leste do Paraguai (SILVANO, 2010). Em função da sua ampla distribuição, é listado como pouco preocupante. Essa espécie é muito comum em áreas antropizadas, mas têm hábitos florestais (HERPETO.ORG, 2015). A reprodução dá-se em riachos e em água parada, como lagoas e poças, de agosto a janeiro, em grandes cordões gelatinosos com milhares de ovos escuros. Os girinos são pequenos, livre-nadantes, permanecem em congregações e se alimentam de matéria em suspensão e na superfície de pedras e plantas submersas (HERPETO.ORG, 2015).

Poços de Caldas é uma cidade localizada na região sul de Minas Gerais, na divisa com o estado de São Paulo, na borda ocidental da Serra da Mantiqueira, fragmento de bioma mata atlântica, considerada a segunda maior floresta pluvial tropical do continente americano e o bioma brasileiro mais alterado pela ação antrópica (LEDERMAN; PADOVAN, 2005) e, mesmo considerado um dos biomas mais ameaçados do mundo, a mata atlântica é considerada um hotspot mundial, sendo uma das áreas mais ricas em biodiversidade (MYERS et al., 2000).

De acordo com quatro levantamentos da herpetofauna no município (GIARETTA; SAZIMA, 1993; LOIOLA, 2010; MONTEIRO-LEONEL, 2004), Poços de Caldas apresenta uma riqueza de 40 espécies de anfíbios, sendo três endêmicas da região de Minas Gerais. No município, as culturas do café e da batata são as mais difundidas, o glifosato é o agrotóxico mais utilizado e muitos corpos d'água utilizados como sítios reprodutivos dos anfíbios anuros coincidem com as áreas agrícolas na região.

Pela necessidade de medidas preventivas e corretivas para a proteção da fauna e da flora, são essenciais os estudos ecotoxicológicos, a fim de estabelecer limites permissíveis de poluentes presentes nas águas.

\section{OBJETIVOS}

Este trabalho teve como objetivo avaliar, sob condições laboratoriais, a toxicidade aguda de formulado comercial com princípio ativo glifosato, analisando a sensibilidade de girinos de Physalaemus cuvieri e Rhinella icterica.

\section{METODOLOGIA}

Foram coletadas desovas de Physalaemus cuvieri e Rhinella icterica (para que os testes fossem realizados com indivíduos de tamanhos e idade semelhantes), as quais foram imediatamente levadas ao laboratório, onde foram colocadas em bandejas de plástico com capacidade para 16 litros, previamente desinfetadas, contendo 4 litros de água declorificada cada. Pouca água de campo foi adicionada às bandejas, para que os girinos eclodissem na mesma água em que fossem submetidos aos tratamentos.

Cada bandeja foi areada com compressor de ar de aquário por $24 \mathrm{~h} \mathrm{dia}^{-1}$, onde as larvas eclodiram. Após a eclosão, as larvas foram alimentadas uma vez ao dia até o início dos tratamentos, no estágio 25 de desenvolvimento (GOSNER, 1960). Os girinos foram alimentados com ração comercial para peixe (Alcon Basic).

Para preparação dos experimentos, foi realizada uma solução mãe de 1.000 ppm em concentração nominal do formulado comercial líquido de composição: sal de isopropilamina de fosfonometil $(\mathrm{N})$ glicina 480 g. $\mathrm{L}^{-1}\left(48,0 \% \mathrm{~m} \cdot \mathrm{v}^{-1}\right)$ - , equivalente ácido de $\mathrm{N}$ glicina (glifosato) 360 g.L $\mathrm{L}^{-1}\left(36,0 \% \mathrm{~m} . \mathrm{v}^{-1}\right)$ - e ingredientes inertes - 684 g.L $\mathrm{L}^{-1}\left(68,4 \% \mathrm{~m} . \mathrm{v}^{-1}\right)$. Para avaliar a sensibilidade das espécies e definir as concentrações a serem utilizadas nos tratamentos, foi realizado um teste piloto com cada uma das primeiras para determinar a menor concentração do formulado comercial que fosse letal para 100\% dos indivíduos em 48 horas de exposição. A menor concentração letal apresentada nos testes piloto foi determinada como concentração máxima utilizada nos tratamentos, reduzindo igualmente em 5 até chegar ao tratamento controle (sem adição do agrotóxico). Os experimentos foram realizados em 48 e 96 horas.

Para cada unidade experimental, 5 girinos foram aleatoriamente transferidos das bandejas para potes de vidro contendo 1 litro de água declorificada + a concentração da solução mãe em seus respectivos tratamentos. Durante o período experimental, os animais não foram alimentados.

Para análise de toxicidade aguda, a presença de mortalidade foi registrada diariamente e os animais mortos foram retirados, registrados e fixados em mistura de 50\% álcool a 70\% e 50\% formol a $15 \%$. Os animais que sobreviveram a cada tempo experimental foram fixados em seus respectivos tempos e tratamentos para análises posteriores. Quatro réplicas foram realizadas para cada grupo de tratamento e controle.

A permissão de coleta, transporte e manutenção temporária dos animais descritos neste projeto foi concedida pelo Instituto Chico Mendes de Conservação da Biodiversidade (ICMBio), órgão do Ministério do Meio Ambiente (MMA), autorização nº 41955-1, de 17 de novembro de 2013. Os experimentos realizados foram aprovados pela Comissão de Ética no Uso de Animais (CEUA) da Universidade Federal de Alfenas (protocolo $\mathrm{n}^{\circ}$ 23087.006836/2013-33, de 10 de setembro de 2013) e as coletas de $P$. cuvieri e R. icterica foram realizadas em 10 de dezembro de 2013 e 22 de outubro de 2014, respectivamente.

Para avaliação dos efeitos subletais, foram realizadas análises do comportamento natatório e morfológicas por meio de exames estatísticos.

Alterações nas respostas comportamentais foram registradas em todos os tempos experimentais (48 e 96 horas), agitando a água de cada tratamento com um bastão de vidro suavemente, por 5 segundos, até formar um redemoinho e observando por 1 minuto a atividade de natação de cada organismo em seu respectivo tratamento (DAVID et al., 2012). Foram analisados o nado normal, quando o animal nadava normalmente e ativamente contra a corrente criada, e o nado afetado, quando o animal nadava um pouco contra a corrente e se deixava levar por ela por tempo superior ao do nado, nadava com o corpo na lateral ou fora do padrão e quando havia paralisia na natação e o animal se deixava levar por completo pela corrente criada. 
As análises morfológicas (morfometria e anormalidades) foram realizadas com os animais fixados que sobreviveram aos tratamentos nos tempos de 48 e 96 horas. Não foram realizadas análises morfológicas nos animais mortos. Foram avaliados os girinos sobreviventes aos tratamentos, pois a inserção caudal alterada e/ou cauda torta podem alterar a mobilidade do animal e, com isso, deixá-lo suscetível ao ambiente onde está inserido; a cavidade oral, quando alterada, pode prejudicar a alimentação dos girinos e, com isso, afetar a sua capacidade de sobrevivência. De acordo com Bahia (2007), os intestinos de girinos herbívoros e onívoros apresentam longos comprimentos, dispostos em dupla espiral, ocupando a maior parte da cavidade visceral, e a malformação desse órgão pode comprometer o desenvolvimento adequado dos animais. Todos os indivíduos submetidos às análises morfológicas foram medidos e pesados.

Para avaliação de malformações, foi utilizada lupa LEICA S8 APO, onde foram realizadas as imagens em câmera de vídeo digital LEICA DFC295 e foram avaliadas a posição caudal (corpo), a condição do intestino (quando visível) e a cavidade oral.

Para avaliação de comprimento dos girinos, foi utilizado papel milimetrado $(1 \mathrm{~mm} \times 1 \mathrm{~mm}$ ) abaixo da placa de petri, por causa do pequeno tamanho dos animais e da dificuldade de utilizar paquímetro digital nessas condições. Para pesagem, foi utilizada balança digital modelo AY220, com precisão de 0,0001 g. O excesso de água dos girinos foi retirado com papel antes da pesagem.

Todos os indivíduos que sobreviveram aos tratamentos foram fixados em solução de $50 \%$ de álcool a 70 e $50 \%$ de formol a $15 \%$, para facilitar o manuseio dos girinos nas análises morfológicas.

Os valores de toxicidade aguda (LC50) para 48 e 96 horas de exposição foram calculados utilizando o software Trimmed Spearman-Kaber Method, versão 1.5 (TSK, 1991).

Para análise dos dados, foram utilizados modelos lineares generalizados (GLM, na sigla em inglês) ajustados à distribuição adequada dos dados. Todas as análises foram realizadas utilizando o software $\mathrm{R}$ 3.11 (R CORE TEAM, 2014).

\section{RESULTADOS}

\section{Testes de toxicidade aguda}

Para Physalaemus cuvieri, as concentrações da solução mãe definidas no teste piloto foram $0 ; 3,19 ; 6,36 ; 9,51 ; 12,64 ;$ e 15,75 mg.L.1 (controle, C1, C2, C3, C4 e C5, respectivamente). No tempo de 48 horas, a mortalidade foi de $0 ; 1 ; 6 ; 20 ; 20$; e 20 indivíduos $(0,5,30,100$, 100 e 100\%); e, no tempo de 96 horas, a mortalidade foi de $0 ; 1 ; 5 ; 17 ; 20$; e 20 indivíduos $(0,10,25,85,100$ e 100\%). A LC50 calculada para o tempo de 48 horas foi de 6,61 mg.L $\mathrm{L}^{-1}\left(5,81\right.$ a 7,53 mg.L $\left.\mathrm{L}^{-1}\right)$ e de 7,12 mg.L. $\mathrm{L}^{-1}(6,21$ a 8,16 mg. $\mathrm{L}^{-1}$ ) para o tempo de 96 horas
Para Rhinella icterica, as concentrações utilizadas foram 0; 4,97; 9,90; 14,78; 19,61; e 24,39 mg.L.' (controle, C1, C2, C3, C4 e C5, respectivamente). No tempo de 48 horas, a mortalidade foi de $0 ; 0 ; 0 ; 9$; 19; e 20 indivíduos (0, 0, 0, 45, 95 e 100\%); e, no tempo de 96 horas, a mortalidade foi de $0 ; 0 ; 5 ; 17 ; 20$; e 20 indivíduos $(0,0,25,85,100$ e $100 \%)$. A LC50 calculada para o tempo de 48 horas foi de $14,78 \mathrm{mg} . \mathrm{L}^{-1}$ (13,65 a $\left.16,01 \mathrm{mg} \cdot \mathrm{L}^{-1}\right)$ e de $11,11 \mathrm{mg} \cdot \mathrm{L}^{-1}\left(9,97\right.$ a $\left.12,68 \mathrm{mg} \cdot \mathrm{L}^{-1}\right)$ para o tempo de 96 horas.

\section{Análise de comportamento natatório}

Para Physalaemus cuvieri, pôde-se perceber que houve redução na frequência de nado normal em relação ao controle conforme as concentrações aumentavam nos dois tempos amostrais. Em 48 horas, $80 \%$ dos indivíduos do controle apresentaram comportamento de nado normal, porém, em C2 (maior concentração na qual permaneceram girinos vivos para análises de comportamento), apenas $43 \%$ dos indivíduos apresentaram comportamento de nado normal. Em 96 horas, apenas $10 \%$ dos indivíduos do controle apresentaram nado afetado, enquanto $87 \%$ deles demonstraram comportamento afetado em C2. Em C3, sobreviveram apenas três indivíduos: dois deles manifestaram comportamento afetado e um, comportamento normal. Não houve diferença significativa em relação ao comportamento afetado e o aumento da concentração no tempo de 48 horas ( $\mathrm{p}=0,2935$ e F = 1,2527). Em 96 horas, houve uma diferença significativa em relação ao nado errático e às concentrações $\left(\mathrm{p}<0,001\right.$ e $\left.\mathrm{X}^{2}=83.335\right)$, para os quais $\mathrm{C} 1$ e $\mathrm{C} 2$ se mostraram iguais estatisticamente, diferindo de $\mathrm{C} 3 \mathrm{e}$ controle, que se revelaram iguais entre si (Figura 1).

$\mathrm{Na}$ análise de comportamento natatório de Rhinella icterica, os indivíduos de $\mathrm{C} 1$ apresentaram maior frequência de comportamento de nado normal em relação ao controle, sem adição do agroquímico,

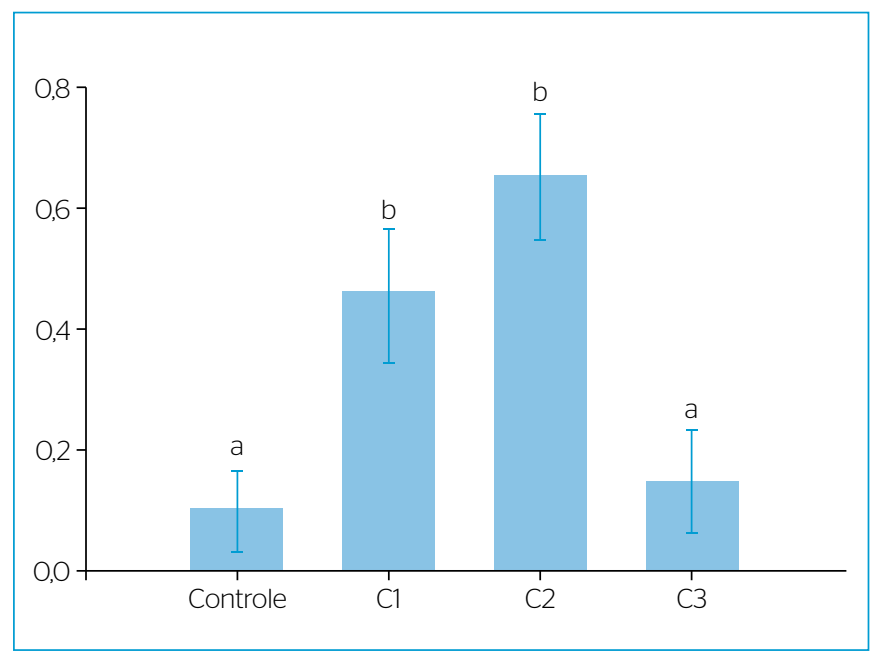

Figura 1 - Relação de comportamento afetado (média de frequência) em relação aos tratamentos de Physalaemus cuvieri no tempo amostral de 96 horas. 
e houve maior incidência de comportamento normal em C2 do que em C1 em 48 horas. Porém, nesse tempo amostral, os indivíduos de C3 demonstraram maior frequência de alteração comportamental em relação à C2. Em 96 horas, houve redução na frequência de comportamento normal em C2 quanto à C1. Não houve avaliação em C4 e $\mathrm{C} 5$, pois todos os indivíduos já haviam morrido no teste de toxicidade aguda. Não foi observada uma relação linear para os comportamentos normais e afetados nos tempos de 48 e 96 horas quanto ao aumento das concentrações, conforme esperado, porém se constatou aumento na frequência de comportamento afetado nas maiores concentrações em ambos os tempos amostrais. Para Rhinella icterica, houve diferença significativa em relação ao comportamento afetado e o aumento da concentração no tempo de 48 horas ( $p=0,01423$ e X² = 92,92). Em 96 horas, não houve diferença significativa entre o comportamento afetado e os tratamentos ( $\mathrm{p}=0,2208$ e $\mathrm{F}=1,5511)$. Apenas $\mathrm{C} 1$ se mostrou diferente significativamente, e controle, C2, C3 e C4 revelaram-se iguais estatisticamente (Figura 2).

\section{Análises morfológicas}

A Figura 3 ilustra o padrão considerado normal de girinos de Physalaemus cuvieri em relação ao corpo e à posição caudal, ao intestino e à cavidade oral.

Physalaemus cuvieri apresentou uma pequena diferença na frequência de indivíduos que sofreram alterações morfológicas entre o controle e $\mathrm{C} 1$ no tempo de 48 horas, e $70 \%$ dos indivíduos do controle foram considerados dentro do padrão normal e $64 \%$ dos de $\mathrm{C} 1$ foram considerados normais. Houve redução para $57 \%$ dos indivíduos considerados normais em C2.

Nas avaliações no tempo amostral de 48 horas, em relação à cavidade oral, 67, 100 e 67\% dos indivíduos de controle, C1 e C2, respectivamente, apresentaram malformações. Apenas os indivíduos do

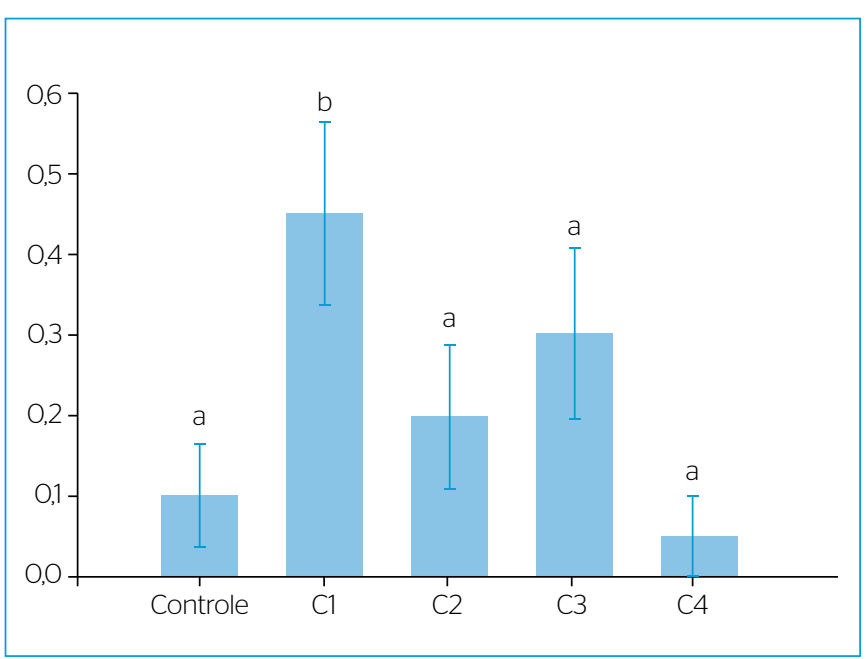

Figura 2 - Relação de comportamento afetado (média de frequência) em relação aos tratamentos de Rhinella icterica no tempo amostral de 48 horas. controle exibiram intestino fora do padrão considerado normal, totalizando $17 \%$, e somente os indivíduos de C2 expuseram corpo com inserção caudal fora de padrão, totalizando $17 \%$. A única alteração em conjunto apresentada nesse tempo amostral foi observada no intestino + inserção caudal, em controle e C2, ambos com 16\% de frequência.

Não houve diferença estatística significativa entre ao aumento das concentrações de glifosato no tocante às análises morfológicas de cavidade oral, inserção caudal e posição do intestino realizada no tempo de 48 horas (inserção caudal $-\mathrm{p}=0,3147$ e $\mathrm{F}=1,18$ ), posição intestinal ( $\mathrm{p}=0,1381$ e $\mathrm{F}=2,0505)$ e cavidade oral $(\mathrm{p}=0,9139$ e $\mathrm{F}=$ $0,9139)$. Também não houve diferença estatística significativa do peso dos indivíduos em relação à concentração $(\mathrm{p}=0,07898$ e $\mathrm{F}=2,6551)$ em 48 horas, porém a diferença foi significativa para o tamanho dos indivíduos ( $\mathrm{p}=0,04786$ e $\mathrm{F}=3,2074$ ), em que o controle se mostrou diferente de C1 e C2, que são estatisticamente iguais (Figura 4).

Em 96 horas, a porcentagem de indivíduos que não apresentaram alterações morfológicas reduziu a $0 \%$ na maior concentração analisada (C3), sendo 50, 50, 13 e 0\% em controle, C1, C2 e C3, respectivamente.

As avaliações de malformações no tempo amostral de 96 horas apresentaram maior frequência de interação entre os fatores avaliados do que apresentado em 48 horas. Observou-se que, assim como em 48 horas, houve incidência maior na alteração morfológica da cavidade oral dos girinos de P. cuvieri, em que 50, 34, 39 e 34\% dos indivíduos de controle, C1, C2 e C3, respectivamente, apresentaram malformações na cavidade oral. Apenas os indivíduos de C3 não apresentaram intestino fora do padrão considerado normal.

No tempo amostral de 96 horas, não houve diferença estatística significativa entre tratamentos e malformação na inserção caudal $(\mathrm{p}=$ 0,2921 e $\left.\mathrm{X}^{2}=70,466\right)$ e na posição intestinal ( $\mathrm{p}=0,693$ e $\left.\mathrm{X}^{2}=83,852\right)$. Porém, houve diferença significativa em relação aos tratamentos e à cavidade oral $\left(\mathrm{p}<0,01\right.$ e $\left.\mathrm{X}^{2}=83,73\right)$. C3 revelou-se diferente estatisticamente de controle, $\mathrm{C} 1$ e C2, que são iguais (Figura 5).

Para Physalaemus cuvieri, em 96 horas, houve diferença significativa tanto no peso quanto no comprimento dos indivíduos, sendo que C3 apresentou-se estatisticamente diferente de controle, C1 e C2 em ambas as variáveis (Figura 6).

A Figura 7 apresenta exemplos de malformações detectadas em relação ao formato do corpo, à inserção caudal e à cavidade oral e no intestino - nestes foram observadas proeminência do fígado e espiral malformada.

Não foi possível analisar a posição intestinal de Rhinella icterica devido a sua coloração escura, que não permitiu a visualização de sua condição. A Figura 8 ilustra o padrão considerado normal de girinos de $R$. icterica em relação ao corpo e à posição caudal.

Rhinella icterica não apresentou uma frequência crescente de malformações em relação ao aumento das concentrações no tempo de 48 horas. A frequência de indivíduos que apresentaram alguma alteração morfológica foi 
de 35, 70, 55 e 55\% em controle, C1, C2 e C3, respectivamente. No entanto, houve uma incidência de indivíduos com malformações após o controle.

Nas avaliações no tempo amostral de 48 horas de R. icterica, houve predominância de malformação da cavidade oral. C1 apresentou maior frequência em $92 \%$ dos indivíduos analisados, enquanto $57 \%$ dos de controle, $81 \%$ dos de C2 e 17\% dos de C3 apresentaram malformação na cavidade oral.
Houve diferença significativa entre as alterações morfológicas de inserção caudal (corpo) ( $\mathrm{p}=0,037$ e $\mathrm{F}=2,6592)$ e da cavidade oral $\left(\mathrm{p}<0,01\right.$ e $\left.\mathrm{X}^{2}=100,35\right)$ em relação aos tratamentos no tempo de 48 horas. Para malformações da cavidade oral, C1 mostrou-se diferente dos outros tratamentos, que se mostraram iguais. Análises de malformação do corpo apresentaram que $\mathrm{C} 3$ diferiu das demais, que também foram similares estatisticamente (Figura 9).

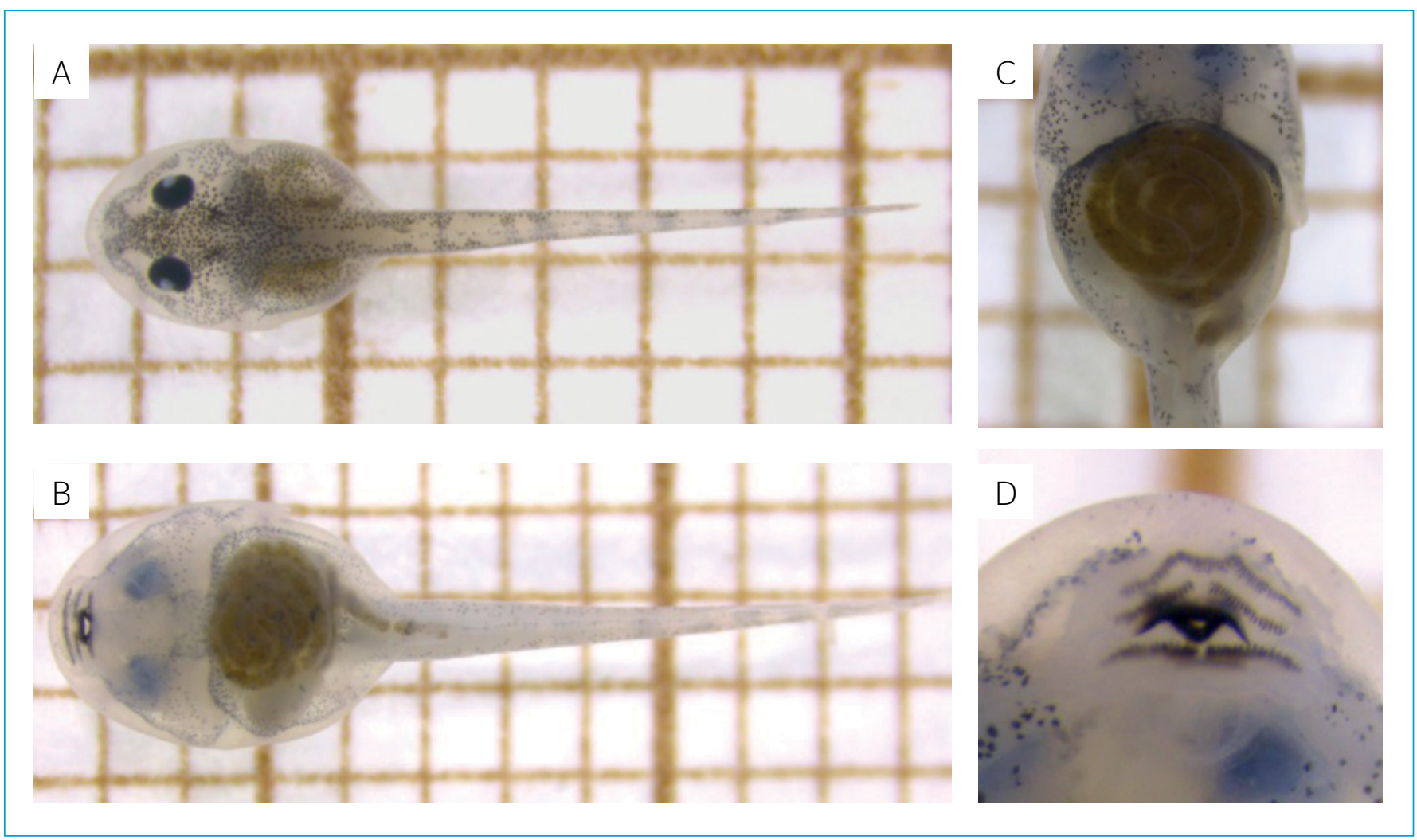

Figura 3 - Padrão considerado normal de girinos de Physalaemus cuvieri em relação (A e B) ao corpo e à posição caudal, (C) ao intestino e (D) à cavidade oral.

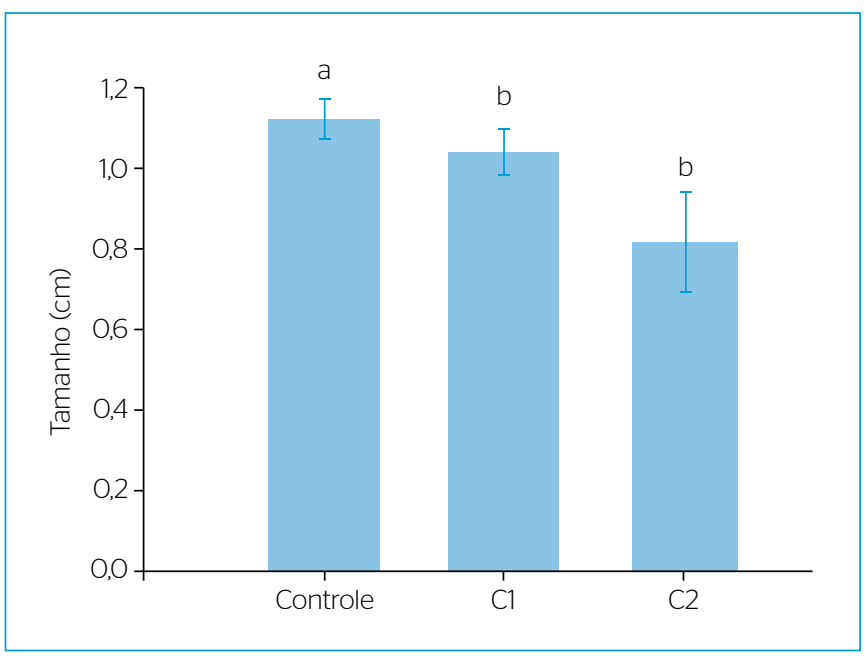

Figura 4 - Relação do comprimento dos indivíduos de Physalaemus cuvieri em relação aos tratamentos no tempo amostral de 48 horas.

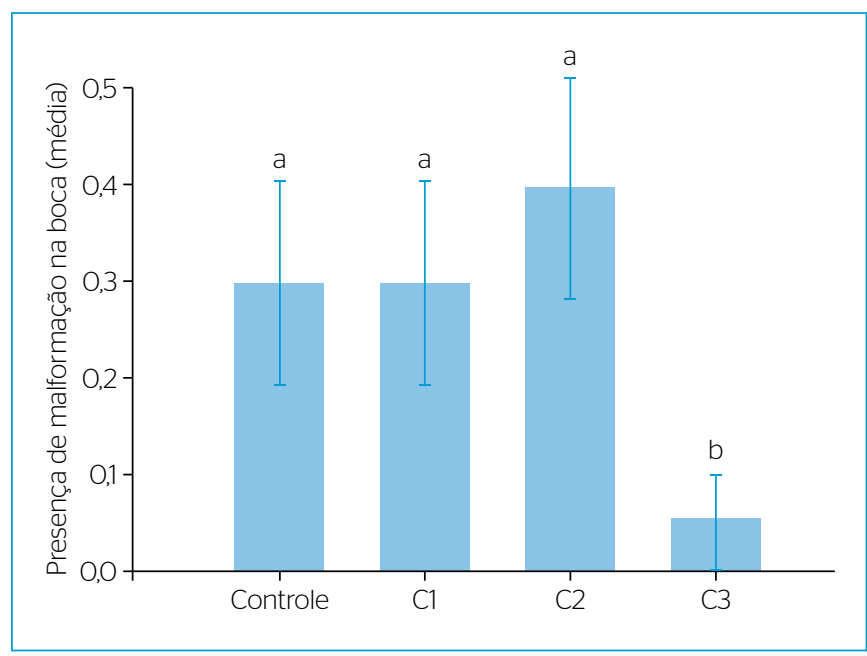

Figura 5 - Relação de malformação da cavidade oral de Pysalaemus cuvieri em relação aos tratamentos em 96 horas. 
Existiu discrepância expressiva do comprimento de Rhinella icterica em 48 horas em relação aos tratamentos, nos quais controle, C1 e C2 foram iguais, porém diferentes de C3, que foi distinto de C4. O mesmo aconteceu com o peso dos indivíduos analisados (Figura 10).

No tempo amostral de 96 horas, a frequência de malformações foi a mesma no controle e em C1, totalizando $45 \%$ dos indivíduos, número que foi reduzido para 33\% em C2 e chegou a 100\% em C3, não apresentando um padrão em relação ao aumento das concentrações, assim como em 48 horas.
Porém, assim como nesse último tempo amostral, no de 96 horas, houve predominância da malformação de cavidade oral, sendo que, dos indivíduos que apresentaram malfomações em controle, C1, C2 e C3, 78, 89, 80 e 67\%, respectivamente, foram na cavidade oral. Houve malformação na posição caudal a partir de C1, e a interação dos fatores foi observada no controle e em C2.

Não houve diferença estatística significativa para malformações da cavidade oral e inserção caudal em relação aos tratamentos para Rhinella icterica no tempo de 96 horas. Houve diferença significativa entre o comprimento de R. icterica e os tratamentos no tempo amostral

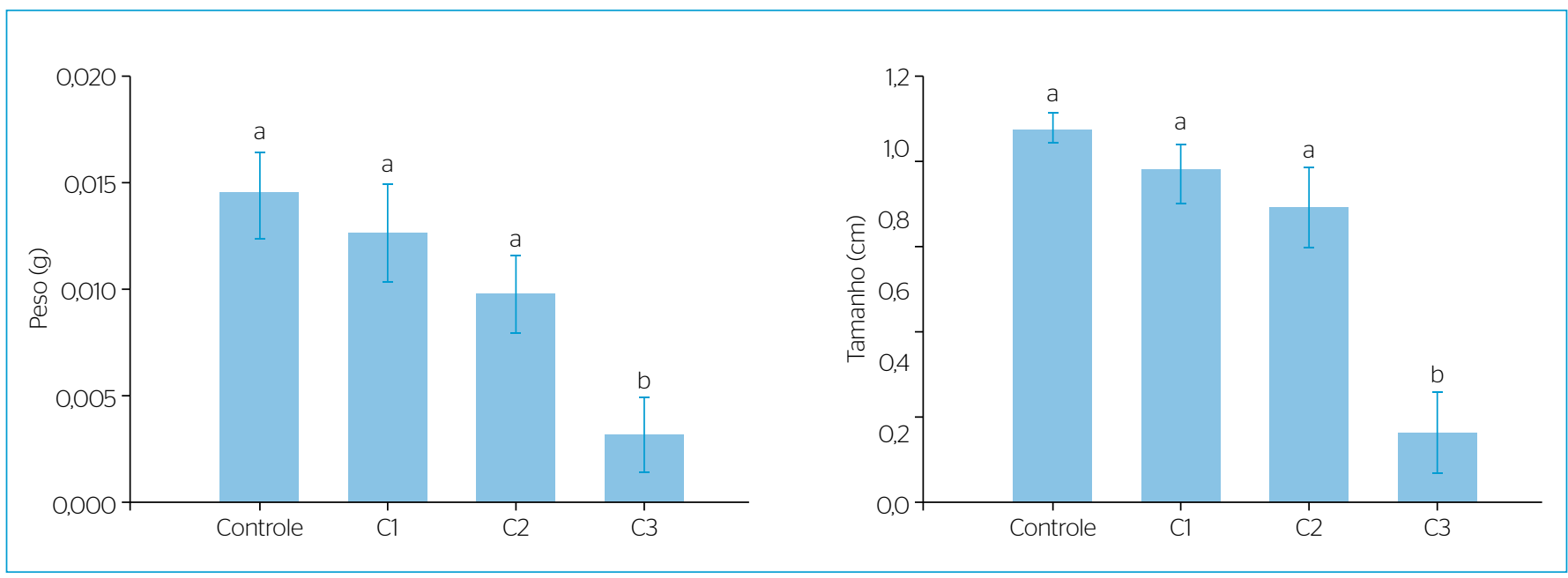

Figura 6 - Relação de peso e comprimento de girinos de Physalaemus cuvier em relação aos tratamentos em 96 horas.
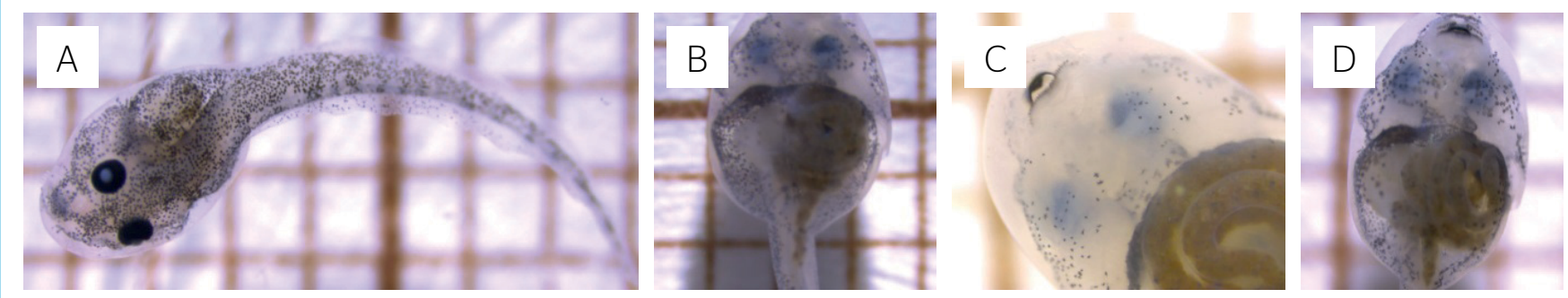

Figura 7 - Physalaemus cuvieri considerados fora de padrão em relação (A e B) ao corpo, (C) à cavidade oral e (D) ao intestino.

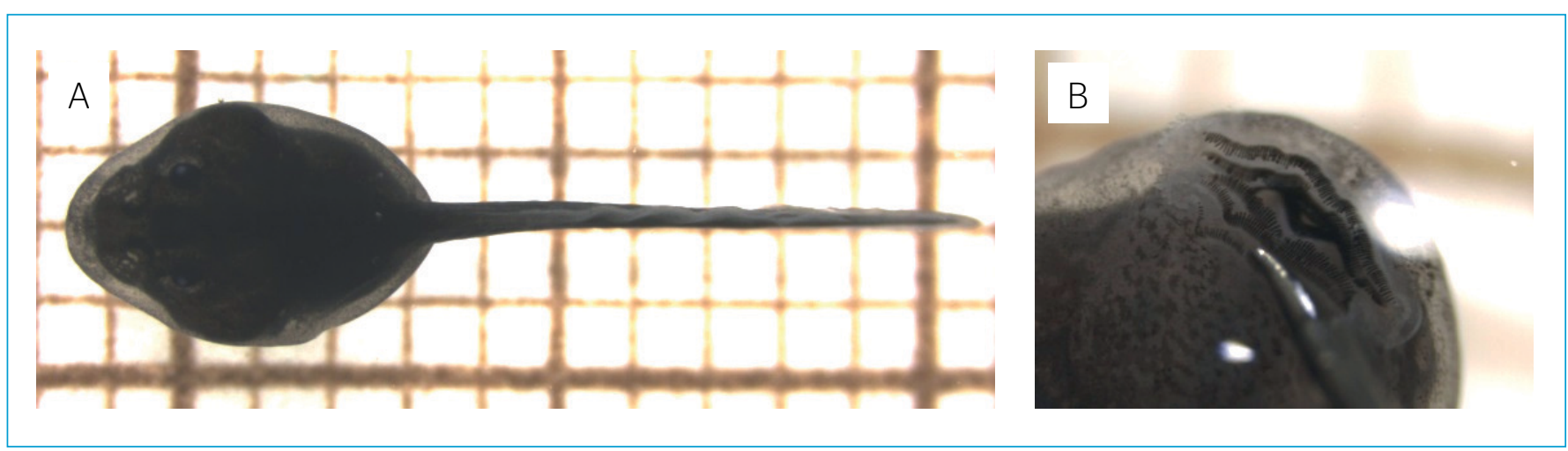

Figura 8 - Padrão considerado normal de girinos de Rhinella icterica em relação ao corpo e (A) à posição caudal e (B) à cavidade oral. 
de 96 horas, em que controle, C1 e C2, iguais estatisticamente, diferiram de $\mathrm{C} 2$, que divergiu de $\mathrm{C} 3$. O mesmo ocorreu com o peso dos girinos em 96 horas (Figura 11).

A Figura 12 mostra exemplos de malformações detectadas em relação ao formato do corpo e à inserção caudal e cavidade oral, cujos dentículos estavam ausentes ou a mandíbula sem completa formação.

\section{DISCUSSÃO}

\section{Teste de toxicidade aguda}

No presente estudo, Physalaemus cuvieri apresentou-se mais sensível ao formulado comercial do que a Rhinella icterica. Em relação a outros organismos avaliados por Tsui e Chu (2003), tanto Physalaemus cuvieri quanto Rhinella icterica se mostraram mais sensíveis à formulação comercial à base de glifosato do que a microalga Selenastrum capricornutum, o protozoário Euplotes vannus e o crustáceo Ceriodaphnia dubia e menos sensíveis do que a microalga Skelotonema costatum e o crustáceo Acartia tonsa, provando a diferença de sensibilidade entre diferentes grupos e a necessidade de se conhecerem os efeitos de compostos químicos em cada grupo animal.

No presente estudo, a LC50 de Physalaemus cuvieri, em 48 horas, revelou-se menor do que em 96 horas, aparentemente significando maior toxicidade em menor tempo. Na margem de $5 \%$ de confiança, os valores encontram-se entre os de LC50 de 7,53 (máxima de LC5048h) e 6,21 (mínima de LC5096h), apresentando igual toxicidade. Tal resultado pode ter sido influenciado pela adsorção do composto químico do tratamento e pela renovação da água em 72 horas sugerida por Walker et al. (2006), influenciando em algum aspecto que não foi possível estimar, devendo ser mais bem analisado em testes posteriores, de preferência sem renovação da água. Outro fator que pode ter influenciado é a carga genética dos indivíduos, que vieram de três diferentes desovas,
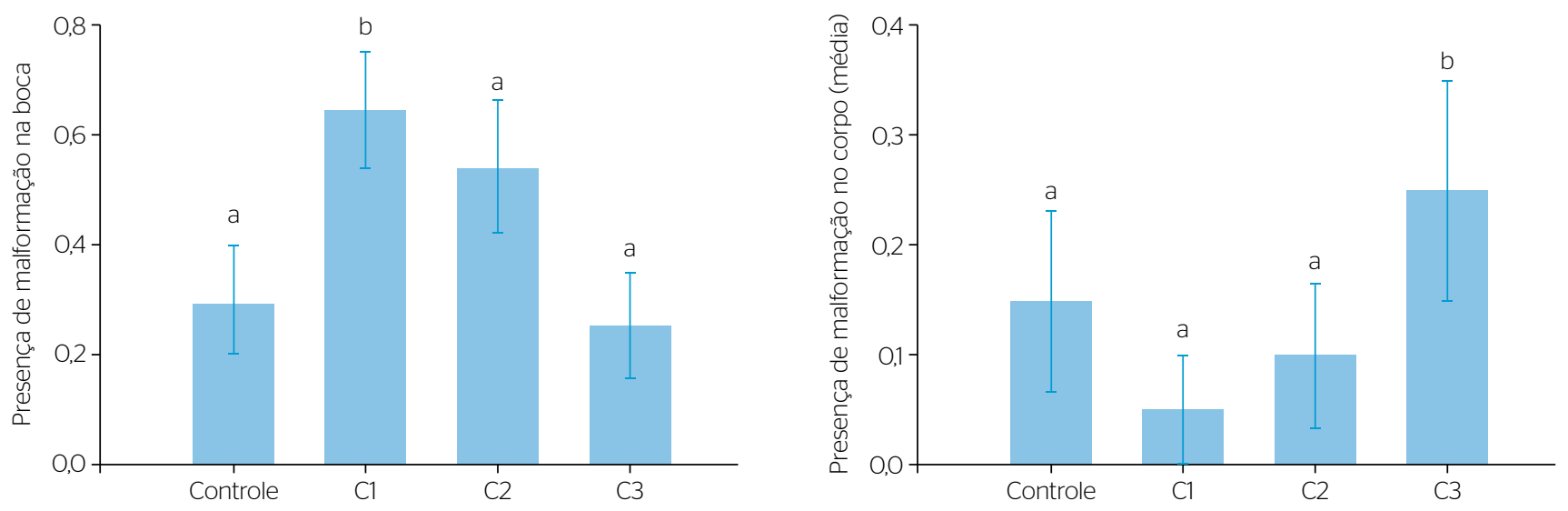

Figura 9 - Relação de malformação da cavidade oral e da inserção caudal (corpo) de Rhinella icterica em relação aos tratamentos no tempo amostral de 48 horas.

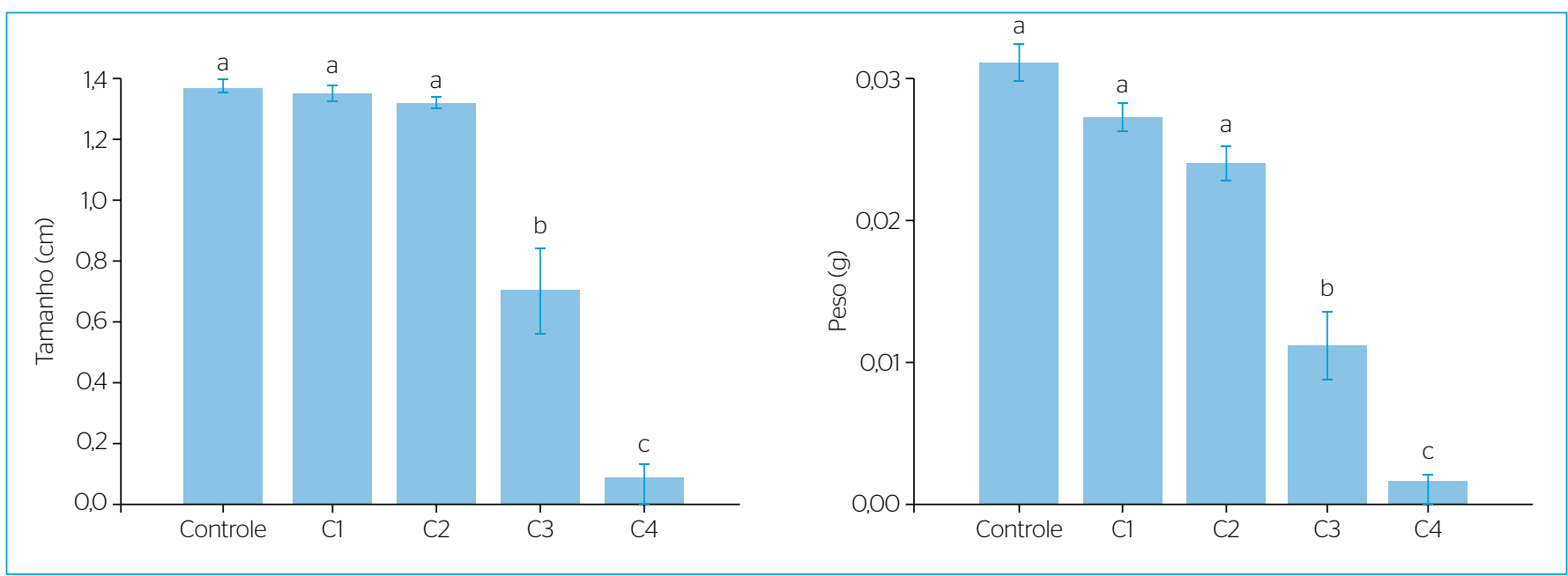

Figura 10 - Resposta estatística no comprimento e peso de Rhinella icterica para controle e as concentrações C1, C2, C3 e C4 no tempo amostral de 48 horas. 
diferente de Rhinella icterica, para a qual todos os indivíduos utilizados nos experimentos foram provenientes de uma única desova. Rhinella icterica apresentou LC50 em 48 horas maior do que em 96 horas, confirmando toxicidade mais alta em maior tempo de exposição ao toxicante. Um fator que pode ter influenciado a diferença de sensibilidade entre as espécies é a discrepância em seu tamanho, sendo a maior espécie mais resistente às perturbações.

Mann e Bidwell (1999) testaram os valores de LC50 em 48 horas para diversos formulados com o princípio ativo glifosato em girinos australianos, e os valores para o formulado comercial variaram entre 8,1 e 32,2 mg. $\mathrm{L}^{-1}$.

Em teste realizado com POEA isolado em Rana clamitans, considerado altamente tóxico, de acordo com a ANVISA (2014), por apresentar LC50 superior a 0,2 até 2 mg.L $L^{-1}$, a LC5024h e a LC5096h foram de 1,1 mg. $\mathrm{L}^{-1} \mathrm{em}$ ambos os tratamentos (HOWE et al., 2004). Howe et al. (2004) concluíram que o surfactante POEA isolado foi o mais tóxico e que as formulações que o utilizam se apresentaram mais tóxicas do que aquelas que continham outras formulações de glifosato.

\section{Análises morfológicas e comportamentais}

O estágio larval dos girinos parece ser um fator responsável pela variação da sensibilidade à toxicidade aguda (DAVID et al., 2012; MANN; BIDWELL, 1999; SEIXAS FILHO et al., 2003). Porém, outros fatores, como idade, peso, dieta, diferenças no manuseio e variações naturais, são parâmetros importantes para determinar as diferenças de sensibilidade entre os animais, porém são pequenas as discrepâncias entre espécies distintas. Adultos e imagos também foram menos sensíveis às formulações de glifosato do que os girinos (MANN; BIDWELL, 1999).

Brunelli et al. (2009), analisando outros estudos, apresentaram que a inibição do crescimento larval é um dos mais sensíveis indicadores de toxicidade no desenvolvimento. Tanto Physalameus cuvieri, quanto Rhinella icterica apresentaram, no presente trabalho, diferenças significativas entre os tratamentos nas variáveis "comprimento" e "peso", o que sugere a toxicidade e a influência da exposição ao formulado comercial nessas espécies.

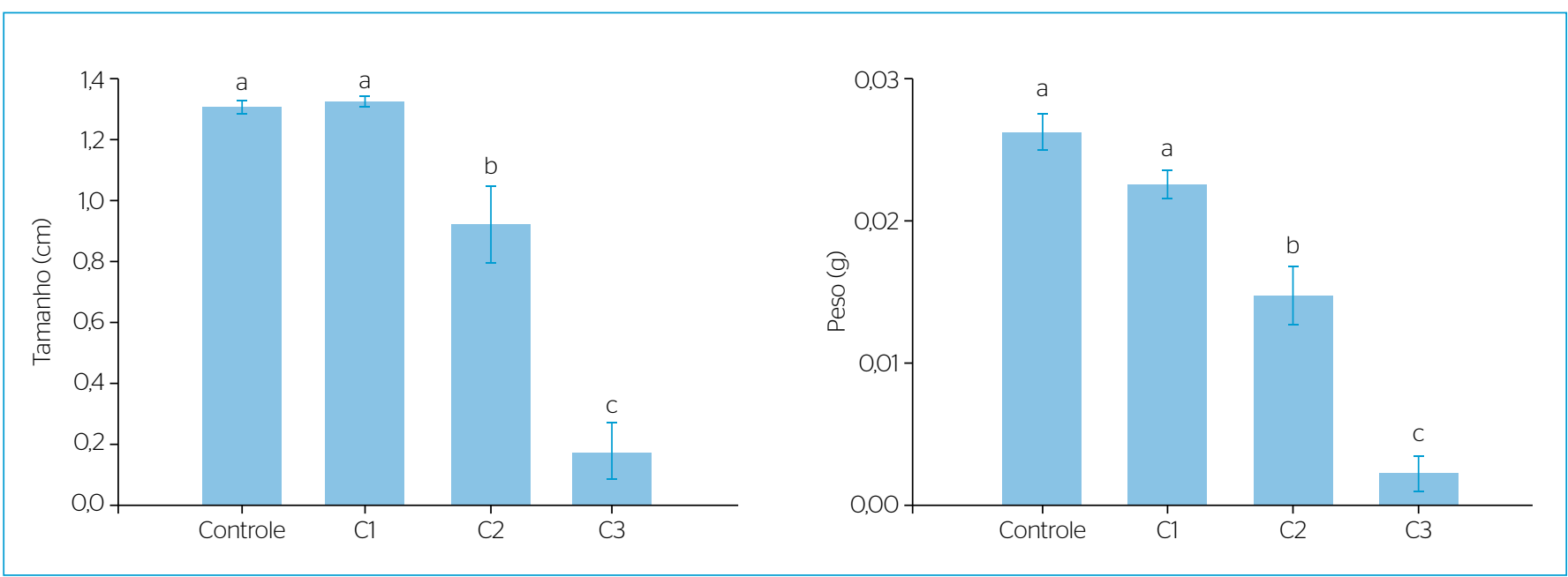

Figura 11 - Resposta estatística no comprimento e peso de Rhinella icterica para controle e as concentrações C1, C2, C3 e C4 no tempo amostral de 96 horas.

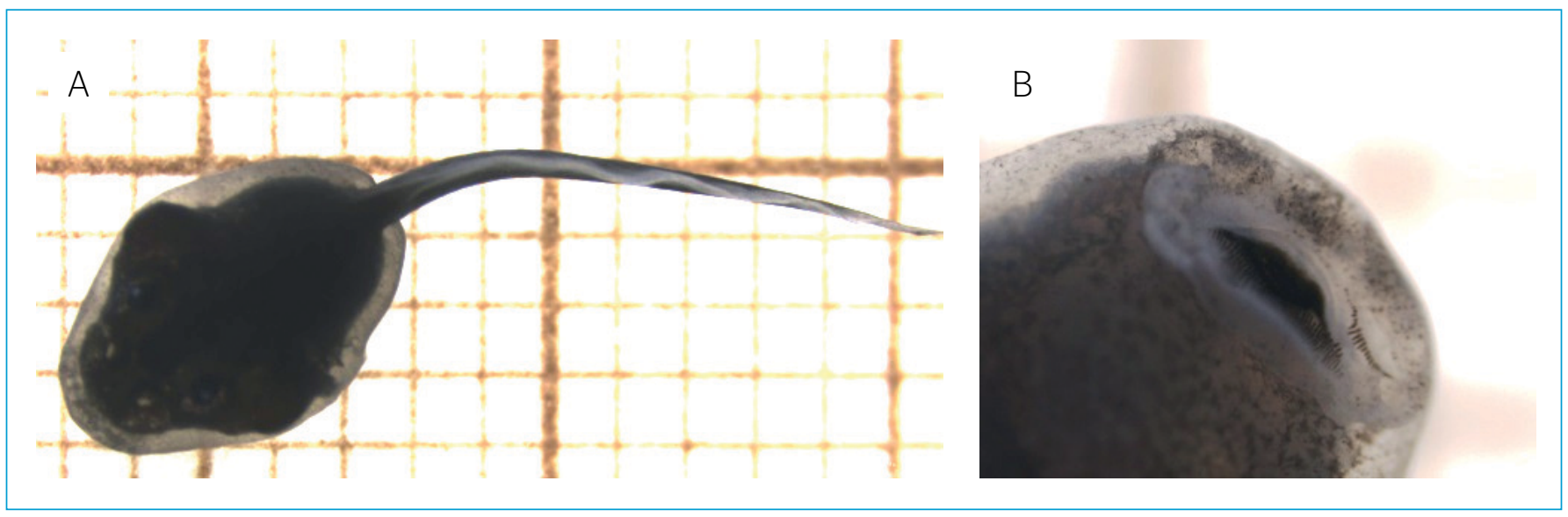

Figura 12 - Rhinella icterica considerados fora de padrão em relação (A) ao corpo e (B) à cavidade oral. 
As análises de malformações apresentadas no presente trabalho estão de acordo com algumas expostas por outros autores, como deformações no disco oral, alterações no nado, despigmentação e cauda curvada (BRUNELLI et al., 2009; LAJMANOVICH; SANDOVAL; PELTZER, 2003). Foi observada alguma alteração em relação ao fígado, que se mostrou proeminente em Physalaemus cuvieri quando houve alteração intestinal. Entretanto, estudos histológicos não foram realizados no presente trabalho e devem ser realizados para concluir que houve efeito provocado pelo agrotóxico em que os animais estavam submetidos.

Características comportamentais são grandes indicadores de sensibilidade a substâncias tóxicas. David et al. (2012) observaram que o comportamento e as malformações morfológicas são sinais típicos de toxicidade.

Não foi detectado, no presente estudo, comportamento natatório dose-dependente, no entanto alterações comportamentais foram detectadas de formas diferentes nas concentrações, podendo ser provocadas pela presença do contaminante.

\section{CONCLUSÃO}

O presente estudo sugere que a aplicação de formulado comercial com princípio ativo glifosato contendo em sua formulação o surfactante POEA tem o potencial de causar mortalidade significativa em girinos de Physalaemus cuvieri e Rhinella schneideri. Este mostrou-se menos sensível que aquele, porém alterações subletais foram detectadas.

No presente estudo, os componentes do formulado comercial não foram analisados isoladamente. Sendo assim, não é possível determinar qual componente ou ação conjunta do composto causou mortalidade nos girinos.

Sugere-se, com o presente trabalho, que o composto em estudo foi responsável pelas alterações morfológicas em peso e comprimento dos girinos de $P$. cuvieri e $R$. schneideri. Propõe-se também que, em elevado tempo de exposição, ele pode afetar a cavidade oral de girinos de Physalaemus cuvieri.

Alterações comportamentais significativas foram observadas em Physalaemus cuvieri e Rhinella icterica expostas a C1 e a C2, no entanto, não foram observadas relações dose-resposta para essas alterações, visto que, nas maiores alterações, não houve efeito significante.

\section{REFERÊNCIAS}

AGÊNCIA NACIONAL DE VIGILÂNCIA SANITÁRIA (ANVISA). (2014) Critérios para a classificação toxicológica. ANVISA. Disponível em: <http:// portal.anvisa.gov.br/criterios-e-exigencias-para-avaliacao-toxicologicade-agrotoxicos-componentes-e-afins>. Acesso em: 3 fev. 2014.

AMARANTE JUNIOR, O.P.; SANTOS, T.C.R.; BRITO, N.M.; RIBEIRO, M.L. (2002) Glifosato: propriedades, toxicidade, usos e legislação. Química Nova, v. 25, n. 4, p. 589-593. http://dx.doi.org/10.1590/ S0100-40422002000400014

BAHIA, V.R.L. de O. (2007) Morfologia e enzimologia do sistema digestório dos girinos de de rã-touro (Rana catesbeiana) durante o desenvolvimento e metamorfose. 164f. Tese (Doutorado em Aquicultura) - Centro de Aquicultura da Universidade Estadual Paulista "Júlio de Mesquita Filho", Jaboticabal.

BLAUSTEIN, A.R.; KIESECKER, J.M. (2002) Complexity and conservation: lessons for the global decline of amphibian populations. Ecology Letters, v. 5, n. 4, p. 597-608. https://doi.org/10.1046/j.1461-0248.2002.00352.x

BLAUSTEIN, A.R.; WAKE, D.B.; SOUSA, W.P. (1994) Amphibian declines: judging stability, persistence and susceptibility of populations to local and global extinctions. Conservation Biology, v. 8, n. 1, p. 60-71. https://doi.org/10.1046/j.1523-1739.1994.08010060.x

BRUNELLI, E.; BERNABÒ, I.; BERG, C.; LUNDSTEDT-ENKEL, K.; BONACCl, A.; TRIPEPI, S. (2009) Environmentally relevant concentrations of endosulfan impair development, metamorphosis and behaviour in Bufo bufo tadpoles. Aquatic Toxicology, v. 91, n. 2 , p. 135-142. https://doi.org/10.1016/j.aquatox.2008.09.006
CONDEZ, T.H.; SAWAYA, R.J.; DIXO, M. (2009) Herpetofauna dos remanescentes de mata atlântica da região de Tapiraí e Piedade. SP, sudeste do Brasil. Biota Neotropica, v. 9, n. 1, p. 157-185. http:// dx.doi.org/10.1590/S1676-06032009000100018

DAVID, M.; MARIGOUDAR, S.R.; PATIL, V.K.; HALAPPA, R. (2012) Behavioral, morphological deformities and biomarkers of oxidative damage as indicator of sublethal cypermethrin intoxication on tadpoles of D. melanostictus (Schneider, 1799). Pesticide Biochemistry and Physisology, v. 103, n. 2, p. 127-134. https://doi. org/10.1016/j.pestbp.2012.04.009

GASQUES, J.G.; BASTOS, E.T.; VALDES, C.; BACCHI, M.R. (2012) Produtividade e crescimento - algumas comparações. Disponível em: <https://bdpi.usp.br/item/002680736>. Acesso em: 29 dez. 2014.

GIARETTA, A.A.; SAZIMA, I. (1993) Nova espécie de Proceratophrys mir. rib. do sul de Minas Gerais, Brasil (amphibia, anura, leptodactylidae). Revista Brasileira de Biologia, v. 53, n. 1, p. 13-19.

GOSNER, K.L. (1960) A simplified table for staging anuran embryos and larvae with notes on identification. Herpetological, v. 16, p. 183190. https://doi.org/10.2307/3890061

GUIMARÃES, E.S.; LACAVA, P.M.; MAGALHÃES, N.P. (2004) Avaliação da toxicidade aguda com Daphnia smilis na água captada no rio Paraíba do Sul e processada na estação de tratamento de água no município de Jacareí - SP - Brasil. Engenharia Sanitária Ambiental, v. 9, n. 2, p. $124-130$ 
HAYES, T.B.; FALSO, P.; GALLIPEAU, S.; STICE, M. (2010) The cause of global declines: a developmental endocrionologist's perspective. Journal of Experimental Biology, v. 213, n. 6, p. 921-933. https://doi. org/10.1242/jeb.040865

HERPETO.ORG. Physalaemus cuvieri. 2014. Disponível em: <http:// herpeto.org/?s=Physalaemustcuvieri>. Acesso em: 12 mar. 2012.

Rhinella icterica. Disponível em: <http://herpeto. org/?s=Rhinellaticterica>. Acesso em: 2 jan. 2015.

HOWE, C.M.; BERRILL, M.; PAULI, B.D.; HELBING, C.C.; WERRY, K.; VELDHOEN, N. (2004) Toxicity of glyphosate-based pesticides to four north American frog species. Environmental Toxicology and Chemistry, v. 23, n. 8, p. 1928-1938. https://doi.org/10.1897/03-71

ITHO, S. de F. Toxicologia. Módulo XII: intoxicações por agrotóxicos. Herbicidas Glifosato. Disponível em: <http://ltc.nutes.ufrj.br/ toxicologia>. Acesso em: 23 jun. 2014.

LAJMANOVICH, R.C.; SANDOVAL, M.T.; PELTZER, P.M. (2003) Induction of mortality in Scinax nasicus tadpoles exposed to glyphosate formulations. Bulletin of Environmental Contamination and Toxicology, v. 70, n. 3, p. 612-618. https://doi.org/10.1007/s00128-003-0029-x

LEDERMAN, M.R.; PADOVAN, M.P. (2005) Conservação da mata atlântica no estado do Espírito Santo: cobertura florestal e unidades de conservação. Programa Centros para Conservação da Biodiversidade-Conservação Internacional do Brasil / IPEMA.

LOIOLA, R.C. (2010) Herpetofauna da Fundação Jardim Botânico de Poços de Caldas, sul de Minas Gerais. 23f. Trabalho de Conclusão de Curso (Graduação em Ciências Biológicas) - Universidade Federal de Alfenas, Alfenas.

MANN,R.M.;BIDWELL,J.R.(1999)Thetoxicity of glyphosateand several glyphosate formulations to four species of southwestern Australian frogs. Archives of Environmental Contamination and Toxicology, v. 36, n. 2, p. 193-199. https://doi.org/10.1007/s002449900460

MCDIARMID, R.W; ALTIG, R. (1999) Tadpoles: The Biology of Anuran Larvae. Chicago e Londres: The University of Chicago Press.

MIJARES, A.; RODRIGUES, M.T.; BALDO, D. (2010) Physalaemus cuvieri. IUCN Red List. Versão 2014.2. Disponível em: <www. iucnredlist.org>. Acesso em: 23 mar. 2014.

MONTEIRO-LEONEL, A.C. (2004) Herpetofauna do planalto de Poços de Caldas, sul de Minas Gerais. 93f. Dissertação (Mestrado em Ciências, na área de Zoologia) - Universidade de São Paulo, São Paulo.
MYERS, N.; MITTERMEIER, R.A.; MITTERMEIER, C.G.; DA FONSECA, G.A.; KENT, J. (2000) Biodiversity hotspots for conservation priorities. Nature, v. 403, n. 6772, p. 853-858. https://doi. org/10.1038/35002501

RELYEA, R.A. (2005) The lethal impact of Roundup on aquatic and terrestrial amphibians. Ecological Applications, v. 15, n. 4, p. 1118-1124 https://doi.org/10.1890/04-1291

RELYEA, R.A.; JONES, D.K. (2009) The toxicity of Roundup Original Max to 13 species of larval amphibians. Environmental Toxicology and Chemistry, v. 28, n. 9, p. 2004-2008. https://doi. org/10.1897/09-021.1

SEIXAS FILHO, J.T.; MELLO, S.C.R.P.; BASTOS, M.P.; MIRANDA, R.G.B.; VEIGA, R.C.A. (2003) Efeito de diferentes salinidades da água sobre o desempenho de girinos de Rana catesbeiana (Shaw, 1802). Augustus, v. 8, n. 16, p. 14-23.

SILVA, H.S.V.P.; LOIOLA, C.; PEREIRA, S.R.F.; SANTOS, R.L.; ANDRADE, G.V.; NUNES, G.S. (2013) Toxicidade aguda e genotoxicidade do agrotóxico comercial Folisuper 600BR a girinos de Physalaemus cuvieri (anura: leiuperidae). Pesticidas, Curitiba, v. 23, p. 1-10.

R DEVELOPMENT CORE TEAM. (2014) $R$ : A Language and Environment for Statistical Computing. Vienna, Austria: R Foundation for Statistical Computing.

SILVANO, D.; SCOTT, N.; AQUINO, L.; KWET, A.; BALDO, D. Rhinella icterica. 2010. IUCN Red List. Versão 2014.2. Disponível em: <www. iucnredlist.org>. Acesso em: 2 jan. 2015. http://dx.doi.org/10.2305/ IUCN.UK.2O1O-2.RLTS.T54668A11184033.en

TSK. (1991) Trimmed Spearman-Karber. Version 1.5. Cincinnati: Environmental Protection Agency.

TSUI, M.T.K.; CHU, L.M. (2003) Aquatic toxicity of glyphosate-based formulations: comparison between different organisms and the effects of environmental factors. Chemosphere, v. 52, n. 7, p. 11891197. https://doi.org/10.1016/S0045-6535(03)00306-0

UNIÃO INTERNACIONAL PARA CONSERVAÇÃO DA NATUREZA (IUCN). (2013) IUCN Red List - The IUCN Red List of Threatened Species, Version 2013.2. Disponível em: <http://www.iucnredlist. org>. Acesso em: 22 ago. 2013.

WALKER, C.H.; HOPKIN, S.P.; SIBLY, R.M.; PEAKALL, D.B. (2006) Principles of ecotoxicology. 3. ed. Boca Raton: Taylor \& Francis Group. 